\title{
LES PAYSAGES NON-FIGURATIFS DE TONY CRAGG
}

\author{
Christian Pierre Kasper ${ }^{1}$
}

Qu'est donc devenu le paysage après deux siècles d'industrialisation? Ou, pour le dire autrement, la partie visible de l'environnement contemporain se laisse-t-elle encore dépeindre comme paysage? Il existe certes des «paysages», naturels ou historiques, devenus de précieuses ressources touristiques pour les pays qui les possèdent, où les éléments qui composent l'espace visible s'ordonnent harmonieusement à un point de vue. Mais, loin de ces «patrimoines de l'humanité», que faire de l'espace vécu de notre expérience quotidienne? Comme le note le géographe Pierre Sansot: «la technologie aura créé un paysage paradoxal dont la plus grande gloire imaginaire aura été de nous mettre au défi de l'imaginer» (Sansot, 1982: 113). Outre la technologie, nous ajouterions le marché, puisque de l'action conjuguée de ces deux forces est née la production de masse. On peut dire que celle-ci, par la multiplication vertigineuse d'objets de toute sorte et de toute taille, du briquet jetable à l'automobile, a bouleversé, en quelques décennies, la visualité de l'espace urbain et suburbain.

Le sculpteur anglais Tony Cragg (né en 1949 à Liverpool) a réalisé, sur une période qui s'étend, grosso modo, du milieu des années 70 au milieu des années 80 , une série d'œuvres qui présente une certaine unité quant aux procédés employés. Ces sculptures se présentent comme des assemblages d'objets trouvés, le plus souvent une grande quantité d'objets usagés ou de fragments d'objets. Outre cette unité, qu'on pourrait dire technique, ces œuvres s'offrent à un type particulier de lecture, qu'on appellera indicielle et qui constitue, c'est là le point de cet article, une de leurs principale originalité. Nous prétendons qu'il s'agit là de «paysages», dans le sens où ces sculptures renvoient à un espace situé hors d'elles-mêmes, l'espace vécu des parcours de l'artiste recueillant les fragments et débris qui les constituent. Des paysages nonfiguratifs, dans le sens où ces «descriptions d'espaces»n'emploient pas les moyens habituels de l'art du paysage, fondé sur le choix d'une «vue» et sur la production d'une ressemblance. Au lieu de cela, Cragg nous montre des indices recueillis dans certains lieux, indices qui nous permettent d'inférer une certaine ambiance visuelle, sans que la

\footnotetext{
${ }^{1}$ Universidade Estadual de Campinas, Brasil.
} 
manière de les présenter ne prétende à quelque similitude avec leur présence dans l'espace où ils ont été collectés.

L'œuvre d'art, comme tout objet matériel, possède un double aspect: d'une part, elle apparaît comme un ensemble de qualités sensibles, couleurs, formes, textures, qu'il est toujours possible d'apprécier pour elles-mêmes; d'autre part, elle se trouve, virtuellement, en rapport avec le reste de l'univers de par les associations qu'elle occasionne. Cette dernière propriété fonde la capacité d'un objet à «tenir lieu de», stands for, dit Peirce, c'est-à-dire à fonctionner comme le signe d'une autre chose, absente. On sait que Peirce a distingué trois modes selon lesquels un objet peut fonctionner comme signe: en vertu d'une ressemblance, d'une convention ou d'une connexion matérielle. Ce dernier cas fonde la catégorie de l'indice qui, selon Peirce: «est un signe qui renvoie à l'objet qu'il dénote parce qu'il est réellement affecté par cet objet» (Peirce, 1978: 140). L'indice, ainsi conçu, peut être rapproché d'une figure de la rhétorique classique, la métonymie, que Fontanier, dans son traité des tropes, définit ainsi: «elle consiste dans la désignation d'un objet par le nom d'un autre objet (...) qui lui doit ou à qui il doit lui-même plus ou moins, ou pour son existence, ou pour sa manière d'être.» (Fontanier, 1977: 79) Remarquons que l'on a ici, comme chez Peirce, l'idée d'un lien existentiel entre les deux entités que le signe met en rapport. On peut donc dire, avec Michel Le Guern, que «la relation métonymique est une relation entre objets, c'est-à-dire entre réalités extralinguistiques; elle est fondée sur un rapport qui existe dans la référence, dans le monde extérieur, indépendamment des structures linguistiques qui peuvent servir à l'exprimer» (Le Guern, 1973: 25). Ce lien existentiel implique que le signe et ce qu'il dénote partagent un contexte commun, une relation qu'il est toujours possible de penser sur le modèle de celle qui lie la partie au tout. C'est évidemment le cas du fragment, qui est reconnu comme tel lorsque l'on évoque l'objet dont il est issu. De même des autres cas de métonymie répertoriés dans les traités de rhétorique: de la cause, de l'effet, de l'instrument, du contenant, de l'agent, etc. Ainsi, comme le résume Verón: «la règle de contiguïté établit une équivalence symbolique entre un ensemble et une de ses parties ou bien entre des parties systématiquement reliées, d’un ensemble donné»(Verón, 1970: 59). On peut opposer ce principe de contiguïté à celui de similitude, comme deux manières distinctes d'enchaîner les idées, ainsi que l'a fait Jakobson dans un article fameux sur l'aphasie (Jakobson, 1963). Identifiant ces deux principes, respectivement, à la métonymie et à la métaphore, Jakobson élargit en outre ces catégories au-delà du langage proprement dit, entre autre 
vers les arts visuels, évoquant les plans métonymiques du cinéma de Griffith. Ainsi, le rapport de contiguïté entre le signe et son objet caractérise aussi bien l'indice, tel que l'a définit Peirce, que la métonymie. On peut voir là les deux faces d'un même mécanisme de signification; lorsque l'on parle de métonymie, on évoque une figure d'expression, alors qu'en parlant d'indice, on pense plutôt à quelque chose qui doit être déchiffré.

On sait que l'acte de communication, ainsi que l'a définit la théorie du même nom, repose sur l'emploi d'un code : l'émetteur se doit d'employer, pour composer son message, des unités connues du destinataire. Ces unités sont donc clairement définies, ce peut être, par exemple, les mots appartenant au lexique d'une langue donnée. En revanche, le rapport de contiguïté qui fonde la métonymie ne désigne pas - au contraire d'un code - une portion de réalité de manière univoque, puisque la même partie peut être partie d'ensembles différents. Comme on le verra, l'ensemble qui comprend la partie examinée doit toujours être inféré et se présente comme probable. C'est dans cette indétermination que réside la possibilité, propre à l'art, nous semble-t-il, d'évoquer des aspects de la réalité qui ne sont pas - ou pas encore - codifiés, c'est-à-dire assujettis aux conventions d'un langage. On a vu que la métonymie, même lorsqu'elle est une figure de langage, porte en fait sur la référence, c'est-à-dire sur une réalité qui est extérieure au langage. En effet, que faut-il pour que nous reconnaissions dans la fumée un signe du feu? Il suffit d'avoir vu un feu brûler et produire de la fumée. Il s'agit là d'une expérience du monde physique qui, en principe, se passe de mot. On imagine donc qu'un être provenant d'une culture complètement différente puisse associer le phénomène «feu» à la perception de la fumée. On voit ainsi que la condition minimale pour qu'une chose fonctionne comme indice ou comme métonymie est l'existence d'une expérience partagée (Lakoff et Johnson, 2003). La métonymie renvoierait ainsi directement à des sensations, à du vécu. Cela ne veut pas dire, évidemment, qu'on en appelle à une expérience originaire, «naturelle», qui précéderait la culture, puisque la possibilité même d'exprimer un sens dépend de l'existence d'un monde commun, et que celui-ci est façonné, de part en part, par une culture. Nous suggérons toutefois qu'il existe une marge, un au deçà du langage, où de nouvelles significations émergent constamment, avant d'être, éventuellement, nommées et classées par le discours commun. C'est en cela que l'emploi de la métonymie (ou de la métaphore, de ce point de vue) comme signe diffère d'un acte de communication: le signe qui est proposé à l'attention du spectateur renvoie à un aspect de l'expérience qu'il peut, ou non, partager 
avec celui qui l'a produit, alors qu'un code institue une règle pour l'interprétation du signe.

Si la similitude glisse sur la surface des choses en un jeu sans fin de renvois, la contiguïté s'étend par inclusion, en une série de contextes emboîtés les uns dans les autres : le fragment d'objet renvoie à l'objet; l'objet (inféré du fragment) renvoie à: son contexte d'usage, à l'ambiance dont il fait partie (un certain univers domestique, par exemple, ou le monde de l'usine), lesquels renvoient à un certain monde géographique, social, historique. C'est donc toute cette chaîne qui se déroule à partir de la considération d'un simple fragment; en effet, si la similitude s'étend en allant du plus semblable au moins semblable, la contiguïté s'étend du particulier au général, ou plutôt en direction d'ensemble toujours plus larges, plus englobants.

En outre, ainsi que le fait remarquer E. Verón, la métonymie ne concerne pas que les objets. Le rapport partie/tout qui la caractérise permet aussi le renvoi à des conduites: le geste s'inscrit dans une séquence de comportement, et c'est sur la base d'une conduite probable qu'il est interprété. Dans le cas qui nous occupe, c'est au faire même de l'artiste - collecter et assembler - que les fragments qui composent l'œuvre renvoient, entre autres choses, bien sûr. Ainsi, si les fragments renvoient à l'objet dont ils ont fait partie, ils renvoient aussi à un univers dont ils sont issus. De même leur présence ici, comme éléments de cette œuvre singulière, renvoie au geste de les ramasser, au lieux où ils ont été trouvés et au parcours au cours desquels l'artiste les a rencontrés, c'est-à-dire à l'expérience sensible de certains espaces.

Pour celui qui regarde, et dans la mesure où il veut y voir des signes, les éléments qui composent ces sculptures se présentent donc comme des indices. Or, comme le savent les lecteurs de romans policiers, les indices doivent être déchiffrés: «que s'est-il passé?» demande-t-on en les interrogeant, à la manière de Sherlock Holmes. Cette lecture des indices est une abduction, et elle consiste à élaborer une hypothèse concernant des faits qui sont donnés. Elle se distingue à la fois de la déduction, qui opère à partir de prémisses certaines, et de l'induction, qui prend acte des régularités de l'expérience, en ce qu'elle opère sur du probable. L'abduction, de fait, conjugue induction et déduction, puisqu'elle infère le probable sur la base de régularités observées. Carlo Ginzburg nous rappelle que ce «savoir indiciel», qu'il définit comme «la capacité, à partir de données apparemment négligeables, de remonter à une réalité complexe» (Ginzburg, 1989: 152) nous vient de nos ancêtres chasseurs, qui ont dû apprendre à déchiffrer les marques laissées par les animaux afin de suivre leurs pistes. 
Quelles sortes de pistes nous offrent alors les objets fabriqués par l'homme? Selon l'anthropologue Alfred Gell:

Tout objet que l'on rencontre dans le monde invite à se demander: «comment cette chose est arrivée ici?». La plupart du temps, les réponses à ce genre de questions vont tellement de soi qu'elles ne jouent aucun rôle dans notre vie mentale consciente. (..) Seuls les géologues, qui ont été formés pour ça, se demandent, en voyant une chaîne de montagne, comment elle est apparue. Mais avec les artefacts, qui sont le produit de types d'agir (agency) dont nous sommes tous capables, la situation est souvent bien différente, et nous nous intéressons effectivement à leur origine (Gell, 1998: 67, ma traduction).

S'agissant d'objets produits par l'industrie, ce n'est pas tant leur origine qui nous intéresse - elle est, pour la plupart d'entre nous, aussi mystérieuse que celle des objets naturels - que l'usage qui en a été fait, leur origine seconde, pourrait-on dire.

Une collection d'indices ne suffit toutefois pas à faire une œuvre. Celle-ci doit trouver une forme. Il semble que cette question ait été abordée de manière singulière dans chacune des œuvres considérées, même si certaines d'entre elles existent en plusieurs versions, déclinant le même principe. A ce point, Il convient donc d'examiner en particulier quelques unes des sculptures appartenant à la période qui nous intéresse.

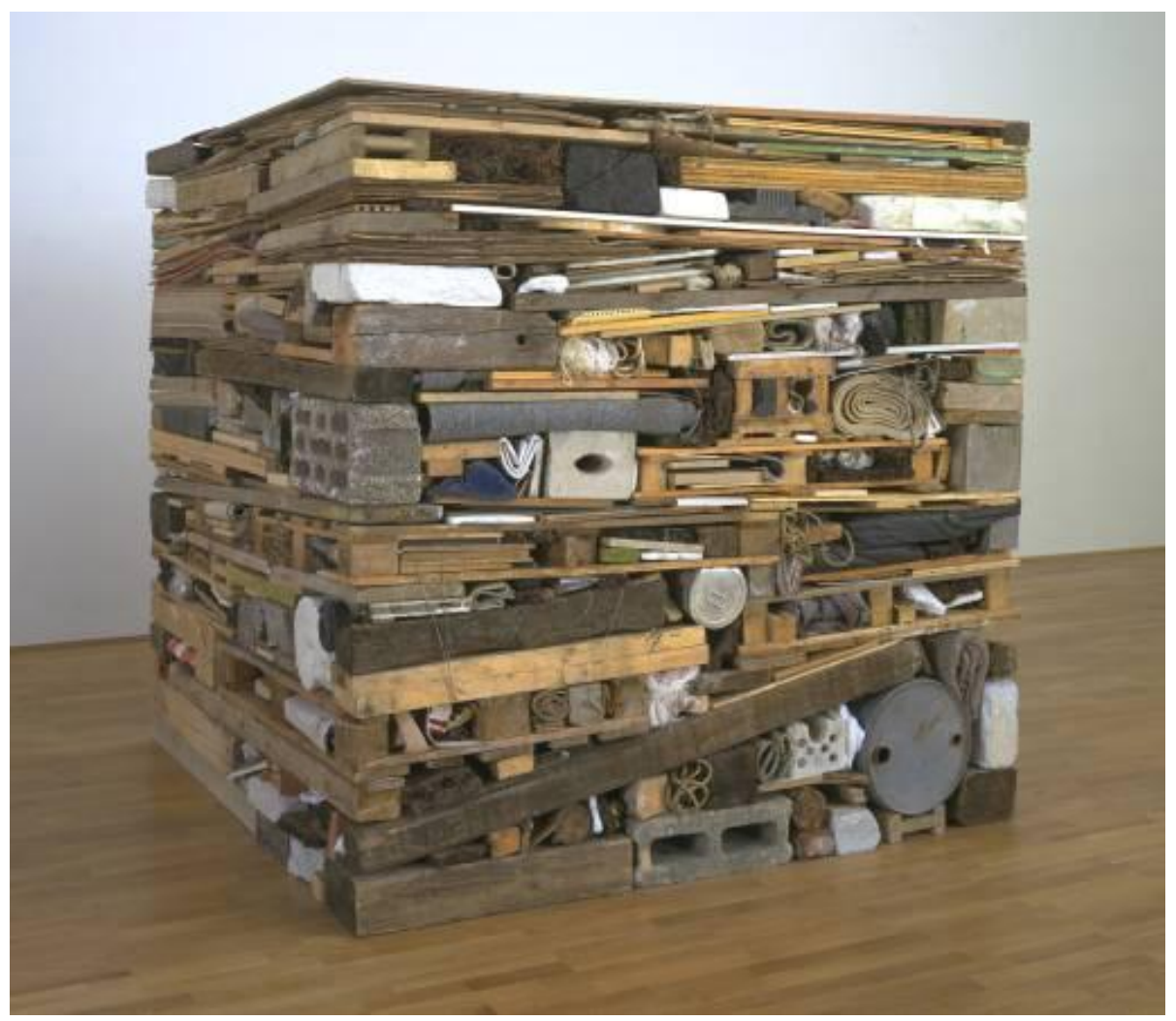

Stack (1975) 
Cette sculpture, qui fait actuellement partie de la collection de la Tate Gallery, semble exprimer plastiquement cette remarque d'Ezio Manzini sur l'environnement artificiel:

Comme le milieu naturel, l'environnement artificiel a une structure géologique. Chaque phase historique y dépose une strate de produits, fruit de ses techniques, de ses formes d'organisation sociale, de ses systèmes de consommation, de sa culture. Des choses disparaissent, d'autres deviennent le substrat de sédimentations futures (Manzini, 1991: 21).

Elle se présente comme une coupe, nette et géométrique, à la manière d'un échantillon qu'on aurait retiré d'une décharge, dans une strate d'artefacts sédimentés, telle que pourrait la pratiquer un archéologue du futur. Réalisée au début de la période qui nous intéresse ici, cette sculpture semble inaugurer la série qui va suivre par quelque chose comme un constat, comme le préambule des arrangements qui vont suivre. Son aspect métaphorique nous semble indiquer une poétique encore à la recherche d'ellemême et qui s'affirmera au cours des années suivantes.

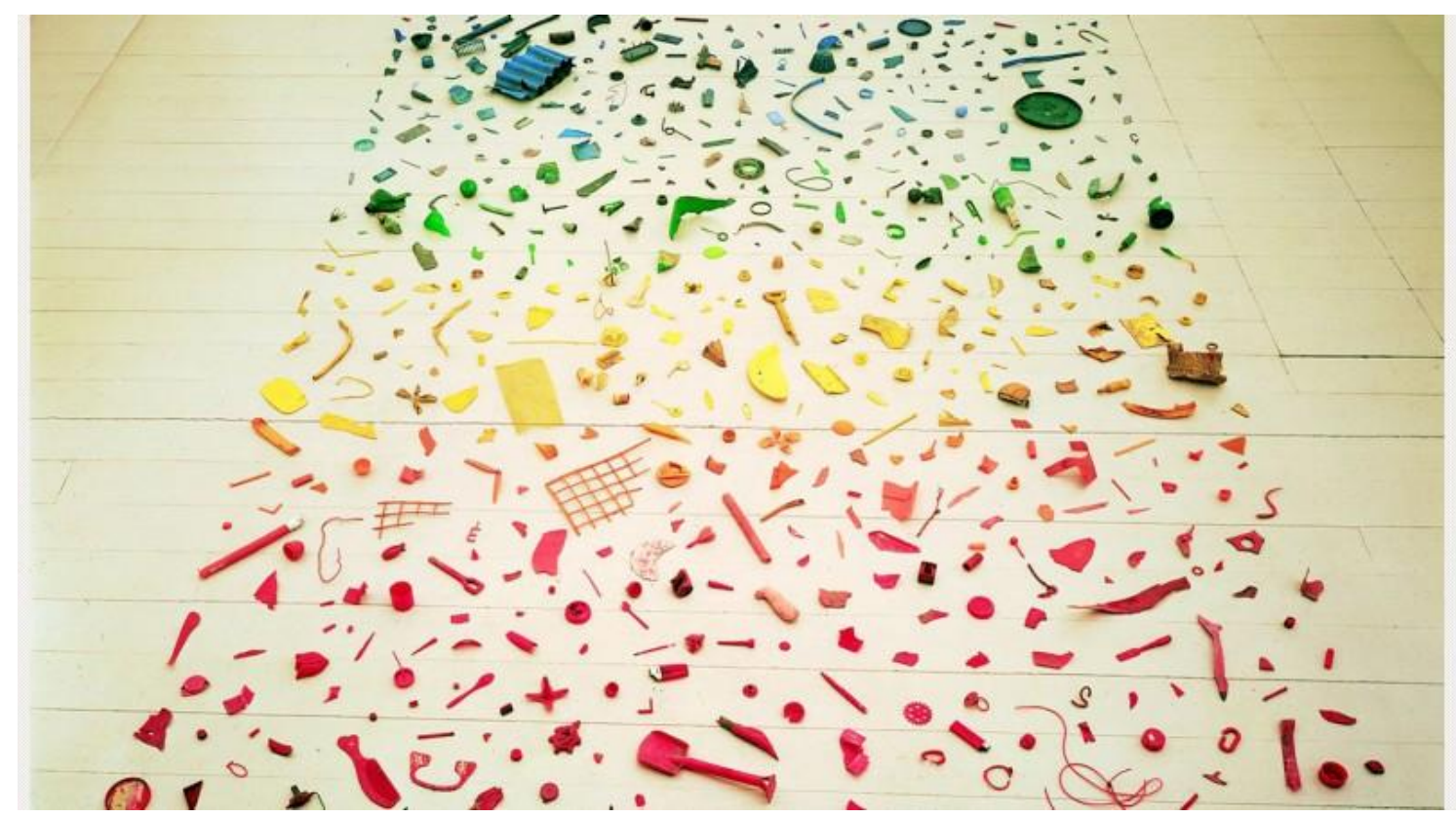

New tone stones, Newton's tones (1978)

Remarquons d'abord le jeu de mot du titre et le rapprochement qu'il établit. Les pierres sont ce que l'on rencontre en cheminant, jonchant le sol immémorial comme ces fragments de plastique parsèment notre chemin, nouvelles pierres du paysage contemporain. On y a vu une allusion aux travaux de Richard Long, éminent 
représentant du Land Art, qui lui réarrange des pierres véritables, rencontrées sur son chemin. Les «pierres» de Cragg sont colorées, elles manifestent, littéralement, toutes les couleurs du spectre, de la manière dont Newton les a ordonnées. Ce placement systématique, semblable à une classification tabulaire, évoque sans doute la méthode scientifique. Il s'en distingue cependant par le souci de la sensation, marque spécifique du travail artistique selon Gilles Deleuze et Félix Guattari:

La composition est esthétique, et ce qui n'est pas composé n'est pas une œuvre d'art. On ne confondra pas toutefois la composition technique, le travail du matériau qui fait souvent intervenir la science (mathématiques, physique, chimie, anatomie) et la composition esthétique, qui est le travail de la sensation (Deleuze et Guattari, 1991: 181).

Dans cette pièce, le recours au spectre des couleurs est une manière de rendre sensible la propriété des matières plastiques de prendre absolument n'importe quelle couleur, à la différence des matériaux traditionnels tels que le bois, la pierre ou les métaux. On reconnaît, parmi les objets et fragments qui composent cette sculpture, une pelle d'enfant, un peigne, des briquets, une cuillère et beaucoup de morceaux dont l'identification est incertaine. On a là comme un tableau de la révolution causée par l'usage massif des plastiques dans le monde des objets, lesquels sont devenus, en quelques décennies, colorés, abondants et jetables. Tony Cragg a repris cette idée de gradation de couleurs dans d'autres pièces, notamment Spectrum (1985), qui présente cette fois une couche compacte d'articles de plastique dont les couleurs sont échelonnée suivant l'ordre du spectre. 


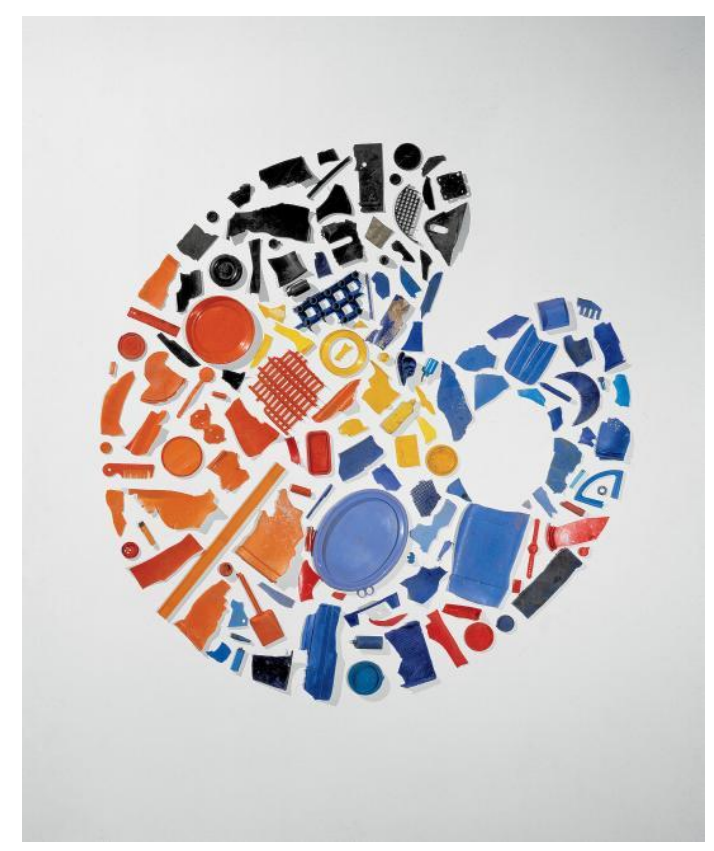

Palette (1984)

On retrouve ici l'ensemble des couleurs du spectre, mais ordonné selon un autre principe. Ce sont ici les couleurs de l'artiste, arrangées dans la forme d'un instrument traditionnel du peintre. Cette association des fragments colorés à un outil de production plastique met l'accent, cette fois, sur le potentiel expressif de ceux-ci : partant de la palette, ils peuvent composer n'importe quelle image. Ce qui est ainsi mis en évidence, c'est le potentiel de recombinaison de ces objets et fragments, qui s'oppose à la pratique commune du recyclage. Celle-ci, en effet, consiste à produire, à partir de déchets, un matériau générique réutilisable, d'où la couleur grisâtre que présentent généralement les plastiques recyclés. Le travail de l'artiste se distingue ici de la rationalité économique, sélectionnant et composant là où celle-ci réduit tout au même dénominateur, ne visant que la continuité de ses propres cycles. 


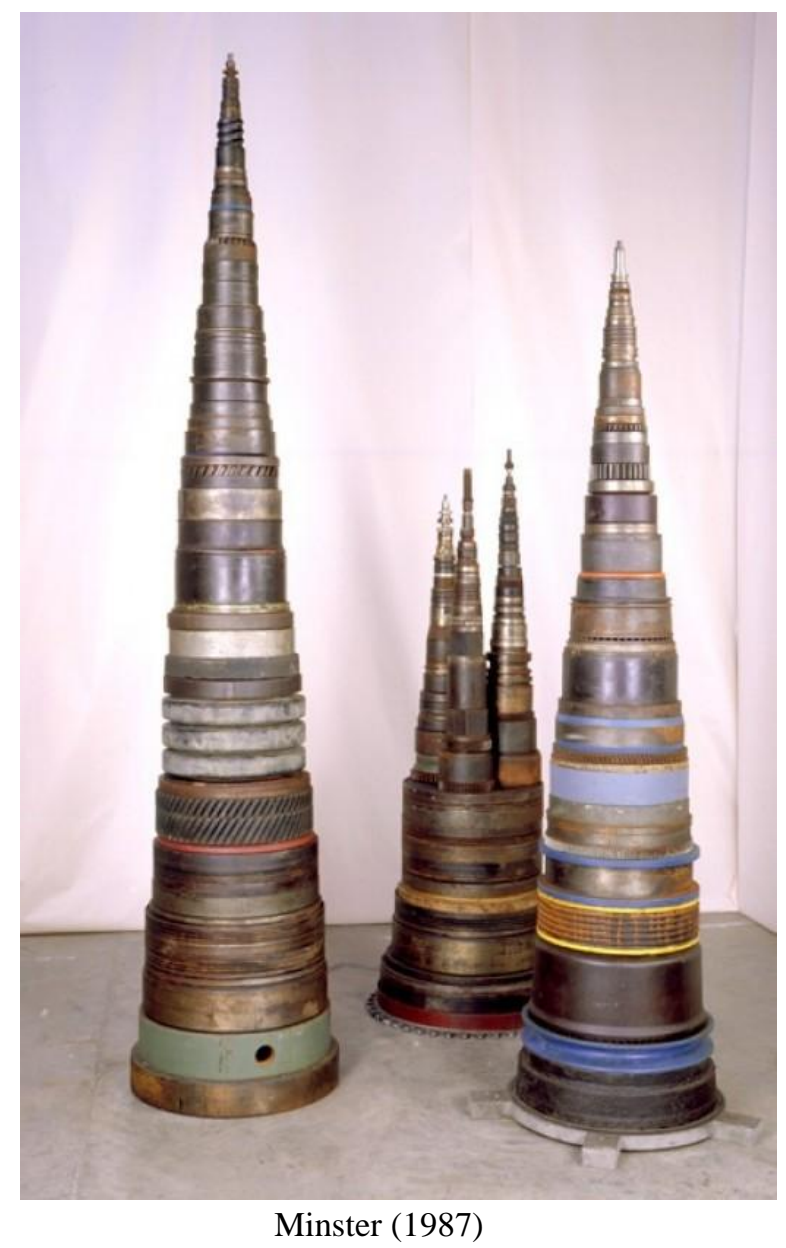

Le travail de mise en ordre concerne ici les dimensions des objets collectés. D'autres œuvres de l'artiste - notamment la série des Axe, qui dessine, par l'étagement d'objets hétéroclites, le volume d'une tête de hache - exploitent le volume des objets plutôt que leur couleur. Dans cette pièce, toutefois, les éléments partagent un trait commun: ce sont tous des cylindres, de longueur et de diamètre variable. Ils sont empilés en échelonnant les diamètres de manière à former des sortes de tours, ce qu'évoque le titre, qui signifie cathédrale. Parmi les objets assemblés, on reconnaît un filtre à air de moteur, un tambour de machine à laver, des roues dentées, des pneus; là encore, nous sommes en présence de restes de la civilisation industrielle. Il y a cependant une différence notable d'avec les sculptures examinées jusque-là: les pièces dont elle est faite ne sont pas des fragments, mais des composants. Le parcours qui va du tout à la partie n'est plus l'accident qui brise, mais une opération de démontage, qui en elle-même induit l'idée de réutilisation. 


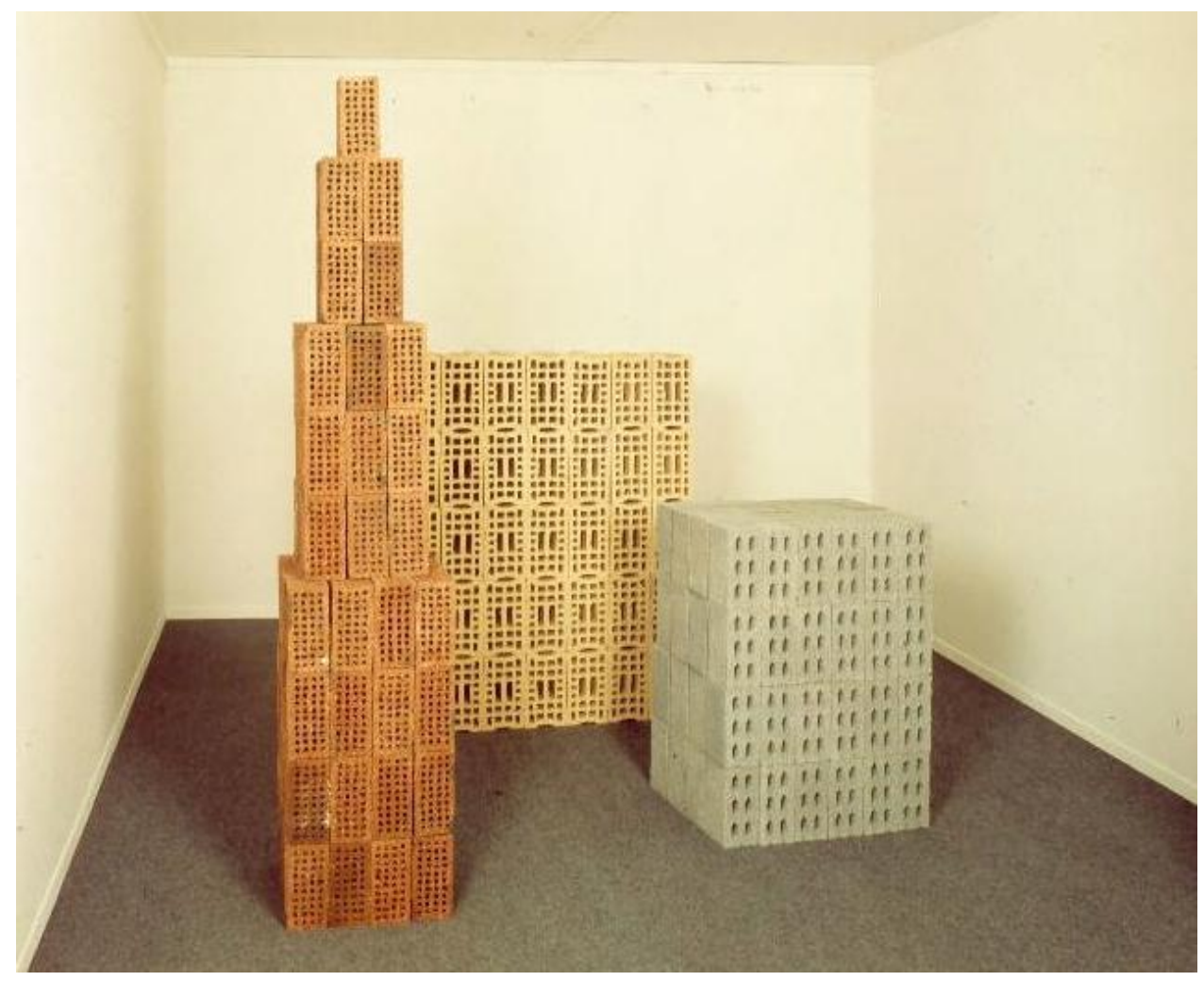

Three modern buildings (1984)

Le signe est ici clairement iconique, puisque la disposition des briques imite la silhouette d'immeubles. Il ne cesse pourtant pas d'être indiciel, puisque c'est avec ces mêmes briques que sont construits les immeubles véritables. L'œuvre ne fait, en quelque sorte, que montrer l'espèce d'écho formel entre le matériau et le produit. L'indice, de fait, a lui-même une forme, puisqu'il doit revêtir une apparence sensible pour pouvoir être perçu ; mais, ainsi que le note Santaella (2000: 131), cette iconicité n'est pas ce qui le définit comme signe : la fumée a bien une apparence visible, une « forme », elle ne ressemble pourtant pas au feu qu'elle dénote en tant qu'indice. Dans cette pièce, la distinction est comme court-circuitée par le fait, certainement contingent, que la forme de la brique répète en miniature celle de l'immeuble qu'elle sert à bâtir.

\section{Passé de l'indice dans l'art}

L'indicialité de l'œuvre d'art n'a longtemps concerné que le rapport de l'auteur à l'œuvre. Il y a d'abord eu la maniera du peintre, marque personnelle de son savoir-faire, qui fonde un style. Puis, au XIX ${ }^{\text {ème }}$ siècle, la curieuse méthode de Morelli, décrite par Ginzburg (1989), qui consistait à identifier l'auteur d'un tableau par la façon de peindre, 
de manière quasi inconsciente, des détails tels que les oreilles des personnages représentés. Il est clair que tant que l'art a employé des techniques comme la peinture, qui transpose les impressions sensibles dans un medium homogène, l'indicialité, en tant que lien matériel ne pouvait lier l'œuvre - en l'occurrence, le tableau - qu'à ses conditions de production, c'est-à-dire principalement à son auteur, puisque les autres facteurs: couleur, toile, pinceaux étaient largement normalisés.

C'est ainsi que l'indice, comme renvoi à une réalité extérieure au processus de la peinture, fait vraiment son entrée dans l'art en 1912, avec les premiers papiers collés de Picasso et Braque. On voit que la démarche de Cragg n'est pas sans antécédents; davantage même que des antécédents, certaines œuvres de l'histoire de l'art inspirent ou provoquent les artistes, suscitent des questions et des problèmes qui peuvent être repris, relancés. Il ne s'agit pas ici d'établir une histoire, de tracer des influences, mais plutôt d'indiquer quelques résonances. Il faut d'abord mentionner Kurt Schwitters, bien sûr, dont les collages Merz témoignent de l'invasion de l'environnement urbain par le texte imprimé. On sait que le mot «merz » est lui-même apparu, au hasard des trouvailles comme un fragment de Kommerz, annonçant déjà la société de consommation à venir. Gilbert Lascault nous présente la démarche de Schwitters comme une relation à l'espace urbain passant par le déchet:

\footnotetext{
Kurt Schwitters (1887-1948) marchait toujours (rapporte Raoul Hausmann) les yeux baissés. Car les restes, les déchets se trouvent le plus souvent au plus bas, jetés, tombés. Il cherchait, tout en marchant, des objets par terre. «Tout lui convenait: tickets de tramway, vieux ou neuf, morceaux de carton, coupures de tissu, boîtes écrasées. Tout était immédiatement classé dans son esprit et destiné à ses collages ». Ce que les autres jettent comme inutile, Schwitters (dès les années 20) le recueille et l’ordonne selon ses désirs artistiques (Lascault, 1979: 365).
}

Plus près de nous, il faudrait aussi évoquer le groupe des Nouveaux réalistes, réunis autour du critique Pierre Restany dans les années 60. De nombreuses œuvres produites par les artistes de ce groupe mettent en jeu des déchets et débris de la consommation de masse et s'offrent à une lecture basée sur l'indicialité. C'est le cas, par exemple, des Accumulations de Arman, des Compressions de César, des Tableaux pièges de Spoerri, ainsi que de certaines œuvres de Martial Raysse, tel l'Arbre bidon, qui semble préfigurer la démarche de Tony Cragg. A propos de l'emploi de ces matériaux, Restany établissait une continuité avec les readymade de Marcel Duchamp, écrivant, dans un manifeste intitulé $A 40^{\circ}$ au-dessus de Dada, que dans le contexte 
actuel, «le ready-made n'est plus le comble de la négativité ou de la polémique, mais l'élément de base d'un nouveau répertoire expressif» (Restany, 1961).

Enfin, certaines sculptures de Tony Cragg peuvent évoquer l'art minimal - nous pensons, par exemple, à certaines pièces de Carl Andre - par la simplicité systématique de leur procédé constructif. Elles s'en distinguent pourtant, précisément par la référence indicielle, toujours présente, alors que les minimalistes ont travaillé à l'effacement de toute référence, à la réduction de l'objet à une présence pure.

On a vu que l'indicialité de l'œuvre renvoyait nécessairement - mais pas exclusivement, bien sûr - à ses conditions de production. Dans cette perspective, ces sculptures de Cragg renvoient doublement à une époque dominée par la consommation de masse. D’une part, et c'est le plus évident, par les matériaux employés, débris de ces articles de plastique bon marché produits par millions et que l'on retrouve partout. D’autre part, le procédé employé renvoie lui-même à deux opérations qui caractérisent la consommation comme pratique: acquérir et combiner (Baudrillard, 1970). Dans le cas de Cragg, ramasser et composer. Dans celui du consommateur, acheter - qui implique toujours de choisir - des articles et les intégrer à un ensemble qui généralement leur préexiste: un vêtement se porte avec d'autres vêtements pour composer une tenue, un meuble s'intègre à un intérieur, un livre à une bibliothèque, un produit de toilette à une routine d'hygiène personnelle, etc. A part quelques cas comme celui de la cuisine, qui implique encore une transformation des produits achetés, l'initiative du consommateur se borne ainsi à combiner des marchandises préalablement choisies. Cragg le fait avec des déchets et, là est le plus important, il invente l'ensemble dans lequel les parties viennent prendre place.

\section{Pour conclure}

Notre titre énonce certainement un paradoxe. Comment, en effet, parler de «paysage» comme forme artistique hors des conventions qui définissent le genre, la figuration en premier lieu? Il nous semble pourtant que les œuvres que nous avons présentées partagent avec le genre paysage, qu'il soit pictural ou photographique, l'évocation de certains lieux; elles décrivent, à leur manière, une certaine expérience du visible. Le rapport que ces œuvres établissent avec les espaces qu'elles évoquent témoigne peut-être d'une impossibilité d'en faire des «paysages» au sens d'image cohérente d'une portion de «pays». On aurait là comme un constat que les lieux produits 
aux marges du monde contemporain - terrains vagues, décharges, zones industrielles plus ou moins abandonnées, etc., ne se laissent pas encadrer comme veduta. L'évocation plastique de ces sortes de lieux doit ainsi se faire par d'autres moyens, et c'est bien ce que nous proposent ces sculptures de Tony Cragg. Mais elles n'en restent pas là : si, d'une part, elles mettent en jeu un effet référentiel au travers des fragments et objets qui, comme on l'a vu, les constituent en signes métonymiques, d'autre part, lorsqu'on les envisage comme compositions, elles apparaissent chacune comme une exploration des potentialités plastiques contenues dans les objets recueillis. On est en somme moins dans la contemplation qu'au coeur de l'action, immergé dans le flux d'objets qui distingue si bien notre époque de l'ère pré-industrielle.

\section{Références}

Baudrillard, J. La société de consommation. Paris: Denoël, 1970.

Deleuze, G., Guattari, F. Qu'est-ce que la philosophie?. Paris: Minuit, 1991.

Le Guern, M. Sémantique de la métaphore et de la métonymie. Paris : Larousse, 1973.

Fontanier, P. Les figures du discours. Paris: Flammarion, 1977 (1830).

Gell, A. Art and agency. Oxford: Clarendon Press, 1998.

Ginzburg, C. Mitos, emblemas, sinais. São Paulo: Companhia das letras, 1989

Jakobson, R. "Deux aspects du langage et deux types d'aphasie". in Essais de linguistique générale. Paris: Minuit, 1963.

Lakoff, G., Johnson, M. Metaphors we live by. The University of Chicago Press, 2003.

Lascault, G. Écrits timides sur le visible. Paris: UGE, 1979.

Manzini, E. Artefacts. Paris: Éditions du Centre Georges Pompidou, 1991.

Peirce, C. S. Écrits sur le signe. Paris: Seuil, 1978.

Restany, P. «A 40 au-dessus de Dada», préface au catalogue de l'exposition Nouveaux Réalistes à la Galerie J, Paris, 1961. Disponible sur: http://mediation.centrepompidou.fr/education/ressources/ENS-nouvrea/ENSnouvrea.htm [consulté le 22/03/2014]

Santaella, L. A teoria geral dos signos. São Paulo: editora Pioneira 2000. 
Sansot, P. «Imaginer le sensible: les paysages technologiques». Traverse, 26 / octobre 1982.

Verón, E. «L’analogique et le contigu». Communication, 15, 1970. 